\title{
Dépérissement des manguiers et citrus dans la région centrale du Togo et méthodes de lutte par des fongicides
}

\author{
Ekanao TEDIHOU1* ${ }^{*}$, Kossi KPEMOUA ${ }^{1}$, Agbéko TOUNOU² \\ ${ }^{1}$ Institut Togolais de Recherche Agronomique (ITRA) \\ 2Université de Lomé - École Supérieure d'Agronomie (ESA/UL), Laboratoire de Recherche sur les Agroressources et \\ Santé Environnementale (LARASE) \\ *Auteur de correspondance: tekanao@gmail.com
}

Original submitted in on 25th September 2017. Published online at www.m.elewa.org on 30 th November 2017

https://dx.doi.org/10.4314/iab.v119i1.1

\section{RESUME}

Objectifs: A partir de 2014 une épidémie de dépérissement de manguiers et d'agrumes s'est déclenchée dans la partie centrale du Togo. Cette étude a été entreprise en vue de déterminer le pathogène responsable et d'identifier un pesticide permettant de lutter contre la maladie.

Méthodologie et résultats: Une prospection a permis la collecte des échantillons de tissu de plantes malades. Les champignons pathogènes potentiels ont été isolés de ces tissus par culture sur PDA et PDA amendé avec des extraits de manguier. Fusarium solani, Phoma glomerata (27,78 et $16,67 \%$ des tissus étudiés) et Lasiodiplodia theobromae ont été isolés mais ce dernier était le plus fréquent et présent dans $83,33 \%$ des tissus étudiés. Cinq fongicides disponibles sur le marché local ont été testés. La symptomatologie de la maladie et la fréquence dans les tissus malades ont permis d'identifier $L$. theobromae comme responsable du dépérissement des manguiers et des agrumes. Tous les fongicides bloquaient la germination des spores de $L$. theobromae. Le fongicide binaire Banko Plus contenant du carbadazim inhibait le mieux la croissance de $L$. theobromae.

Conclusion : La lutte contre l'épidémie se résume au contrôle de $L$. theobromae. Dans un premier temps, l'incorporation du Mancozeb au milieu de culture avant son insémination a été plus efficace suivi dans l'ordre par celle du Callomil, du Jumper, du Callicuivre et du Banko Plus. Ce traitement pourrait permettre la prévention de la maladie par blocage de la germination des spores. Deuxièmement, dans le cas de l'aspersion de suspensions de fongicide sur les colonies en croissance sur PDA, le Banko Plus a donné le meilleur résultat. La combinaison de Banko Plus comme fongicide systémique et du Mancozeb comme fongicide de contact devrait permettre la lutte contre le dépérissement du manguier et des citrus au Togo.

Mot clef : Dépérissement, Lasiodiplodia theobromae, fongicide systémique, Manguier, Citrus. 


\section{Dieback of mangos and citrus in the central region of Togo and control methods by fungicides ABSTRACT}

Objectives: Begun in 2014, an epidemic of mango and citrus dieback broke out in the central region of Togo. This study was undertaken to determine the causal pathogen and to identify a pesticide to control the disease.

Methodology and Results: A survey was conducted to collect tissue samples from diseased plants. Potential pathogenic fungi were isolated from these tissues by cultivating them on PDA and PDA amended with mango extracts. Fusarium solani, Phoma glomerata (27.78 and $16.67 \%$ of the tissues studied) and Lasiodiplodia theobromae were isolated but the later was more frequently encountered and present on $83.33 \%$ of studied tissues. Five fungicides available on the local market were tested. The symptoms of the disease and the frequency in the diseased tissues pointed to $L$. theobromae as the main causal agent. All fungicides blocked the germination of $L$. theobromae spores. Banko Plus, a binary fungicide containing carbadazim, best inhibited the growth of $L$. theobromae.

Conclusions and application of findings: The control of the epidemic can be summarized to the control of $L$. theobromae. Firstly, the incorporation of Mancozeb into the culture medium before its insemination was the most effective followed in order by Callomil, Jumper, Callicuivre and Banko Plus. This treatment blocks spores germination and could be used to prevent the disease. Secondly, in the case of spraying of fungicide suspensions on growing colonies on PDA, Banko Plus gave the best result. The combination of Banko Plus, used as systemic fungicide, and Mancozeb, as contact fungicide, should be effective in controlling the dieback of mango and citrus in Togo.

Key word: Dieback, Lasiodiplodia theobromae, systemic fungicide, Mango tree, Citrus.

\section{INTRODUCTION}

Le dépérissement du manguier et des citrus est connu dans de nombreuses régions productrices de fruits (Al-Sadi et al., 2013) mais son incidence est plus importante dans les zones tropicales et subtropicales (Khanzada et al., 2006;Tovar et al., 2013). L'Afrique de l'Ouest, et donc le Togo, fait partie des zones qui connaissent cette maladie touchant une diversité d'arbres fruitiers. II est plus couramment observé dans la région centre-ouest du Togo sur les manguiers et les citrus. La gravité de la maladie est préoccupante car cette région est justement l'une des principales régions pourvoyeuse des fruits de manguiers et des agrumes pour le Togo. L'incidence de cette maladie varie entre 55 et $80 \%$ et peut causer une mortalité de 20 à $35 \%$ des plants (Haougui., 2013). Le dépérissement du manguier et des citrus est très tôt considéré comme une maladie causée par des champignons (Davis et al., 1987 ; Reckhaus, 1987 ; Meah et al., 1991). II est associé à divers pathogènes dont les plus probables sont Lasiodiplodia theobromae, Fusarium sp. (Khanzada et al., 2004) Phoma sp. et
Hendersonulla toruluoide (Haougui, 2013).Cette pathologie est susceptible de provoquer des dépérissements des plants de manguier ou de citrus. Les symptômes commencent au niveau des feuilles qui nécrosent des bordures vers les nervures principales. Les feuilles ainsi affectées brunissent et meurent en s'enroulant vers le haut. Dans les cas sévères, en moins de deux mois la maladie évolue vers les petites branches des extrémités qui meurent à leur tour et la nécrose évolue vers les branches les plus importantes et au stade final c'est l'arbre tout entier qui peut mourir. Le plus souvent l'arbre, même mort, conserve sa silhouette c'est-à-dire que les feuillent sèches ne tombent pas. Dans de nombreux cas le dépérissement ainsi décrit est concomitant avec une abondante exsudation de gomme dont la couleur varie pour les manguiers du jaune au brun sombre et pour les citrus du jaune transparent au brun. Cette exsudation apparait sur le tronc et sur les plus grosses branches. Dans certains cas sévères les parties extérieures des branches peuvent présenter des fissures et des fentes. 
L'examen d'une coupe longitudinale des branches lignifiées et portant les symptômes de dépérissement présente un brunissement des tissus vasculaires centraux. Plusieurs études ont prouvé que les facteurs, tels le stress hydrique (Desprez-Loustau, et al., 2006) et minéral (Khanzada et al., 2004) tendant à affaiblir les plantes peuvent favoriser l'avènement et l'évolution de cette maladie). Le but de cette étude

\section{MATERIELS ET METHODES}

Trois grandes étapes ont été suivies au cours de cette étude. Dans un premier temps les échantillons susceptibles de livrer les pathogènes responsables de la maladie ont été collectés sur les sites de l'épidémie. Les échantillons ainsi collectés ont été traités au laboratoire pour en isoler et identifier les pathogènes et enfin, toujours au laboratoire, des tests d'efficacité in vitro ont été menés.

Collecte d'échantillons : La collecte d'échantillons s'est faite au cours d'une prospection sur le terrain dans la zone centrale du Togo où l'épidémie a été signalée. Au cours de la prospection les plants de manguier de diverses variétés ont été examinés dans la commune de Bassar ( 5 sites pour 7 échantillons), à Kabou ( 3 sites pour 3 échantillons), à Temèmè sur la route de Kara (2 sites pour 3 échantillons) et à Bandjeli (1 site pour 1 échantillon). L'examen des citrus a été faite à Kabou (1 site: citronnier pour 1 échantillon) et à Sara (1 site: citronnier, pamplemoussier, oranger pour 3 échantillons). Au total 18 échantillons ont été prélevés tous constitués de tissus végétaux (feuilles, tiges) malades. Tous les tissus provenant d'un arbre malade sont regroupés en un échantillon et le nombre d'échantillons par site varie en fonction du nombre d'individus malades ou de la diversité spécifique présente sur le site. Les échantillons ainsi prélevés ont été conservés dans des sacs en plastique et transportés au laboratoire de phytopathologie de l'ITRA à Lomé où leur conservation à longue durée s'est faite à $4^{\circ} \mathrm{C}$. Au cours de la collecte des échantillons l'historique des plants malades était recueilli auprès des propriétaires ou des riverains.

Isolation au laboratoire : La deuxième étape de l'étude s'est déroulée au laboratoire et a consisté en l'isolation des champignons présents au sein des échantillons. La méthode utilisée est basées sur celle de Slippers et Wingfield (2007) avec de mineurs amendements notamment en ce qui concerne l'utilisation de l'infusion de tige de manguier et de est d'isoler à partir des échantillons de plantes malades ces pathogènes, de les identifier et de procéder à des tests d'efficacité d'un certain nombre de fongicides sur les pathogènes identifiés. Ceci dans le but à courts termes de disposer d'un moyen de lutte efficace contre les dépérissements des manguiers et des agrumes au Togo.

PDA/Agar. Pour ce faire, des morceaux de tiges ou de feuilles de manguier ou de citrus d'approximativement 1 centimètre de long ont été superficiellement désinfectés avec une solution à $10 \%$ de l'hypochlorite de sodium puis rincés avec de l'eau distillée stérilisée. Ces fragments de tissus végétaux ainsi superficiellement stérilisés sont ensuite déposés sur du milieu de culture artificiel (PDA) amendé avec de la streptomycine. Les boîtes de Pétri contenant le substrat de culture et ayant reçu les morceaux de tissu superficiellement stérilisés sont incubées à $30^{\circ} \mathrm{C}$ avec alternance de lumière et d'obscurité $(12 \mathrm{H} / 12 \mathrm{H})$. Après 7 jours d'incubation, les boîtes sont examinées et tous les champignons qui se sont développés sur le milieu autour des tissus de bois sont repiqués sur du PDA, sur du PDA amendé avec une infusion de tige de manguier (mPDA) et sur du PDA amandé avec agar à $50 \%$ du poids (PDA50). Les milieux ainsi inséminés sont aussi incubées dans les mêmes conditions que précédemment pour 7 autres jours. Les colonies de champignons qui s'y sont développées ont été alors observées et identifiées.

Identification au laboratoire : La dernière étape consiste en une identification des pathogènes responsables du dépérissement. Pour cette dernière étapes les colonies sont observées macroscopiquement pour voir leur couleur, leur aspect etc., et microscopique pour déterminer la morphologie et les caractéristiques de leurs conidies. L'identification s'est fait en suivant les consignes et les descriptions de divers auteurs (Punithalingam, 1976; Kausar et al., 2009). Une fois l'identification faite les boîtes contenant les colonies sont conservées au réfrigérateur à 4 degrés Celsius pour des tests d'efficacité.

Test d'efficacité : Les tests d'efficacité avaient pour objectif de sélectionner parmi les fongicides homologués et disponibles localement le plus efficace pour le contrôle de la maladie de dépérissement des manguiers et des agrumes. Pour les essais proprement dits le matériel biologique était donc constitué des 
isolats préalablement identifiés et maintenus sur du PDA conservé au réfrigérateur à $4^{\circ} \mathrm{C}$; et les cinq fongicides homologués au Togo utilisés étaient: le Banko Plus (carbendazime et chlorothalonil), le Jumper (chlorothalonil), le Callomil 72WP (métalaxyl et oxyde de cuivre), le Callicuivre (oxyde de cuivre) et le Mancozeb. La méthode utilisée s'est inspirée de celle de Tovar et al. (2013) et a consisté en deux traitements impliquant les cinq fongicides avec deux types de traitement témoins. Le premier traitement consistait à préparer du PDA avec la dose du fongicide recommandé par le fabricant puis à inoculer le substrat ainsi obtenu (PDA + fongicide) avec le champignon. Le deuxième traitement consistait à préparer du PDA, à l'inoculer avec le champignon avant d'asperger les colonies de champignon en développement après deux jours d'incubation avec une suspension aqueuse des fongicides testés à la dose recommandée et selon le mode d'emploi du fabricant. Le troisième traitement

\section{RESULTATS ET DISCUSSION}

L'observation des plants malades et leur historique permettent de déduire que le dépérissement des manguiers concernait surtout les plants ayant un âge assez avancé (10 à plus de 25 ans) et en plus les périodes d'avènement des épidémies coïncidaient avec les fins de saisons caractérisées par des sécheresses sévères advenues dans la zone. consistait au témoin 1 c'est-à-dire que sur du milieu PDA inoculé avec les champignons aucun autre traitement n'est appliqué (témoin absolu). Enfin le quatrième traitement ou témoin 2 reprend le deuxième traitement mais au lieu d'une suspension de fongicide c'est de l'eau distillée et stérilisée qui est aspergée sur les colonies. Chaque traitement était répété trois fois. Les boites de Pétri contenant les différents traitements sont observées quotidiennement à partir du troisième jour après inoculation considéré comme jour 1. Les diamètres des colonies sont mesurés et enregistrés.

Analyse statistiques : Les analyses de variances ont été effectuées en utilisant la fonction ANCOVA de XLSTAT. Les moyennes des diamètres ont été utilisées pour tracer les courbes et ont été comparées entre elles par le test LSD (least significant difference) à $P \leq$ 0,05 . Pour apprécier l'efficacité des fongicides une comparaison de leur effet sur les huit mesures effectuées sur chacun des trois pathogènes a été fait.
Isolation et identification : Trois espèces de champignons susceptibles de provoquer le dépérissement sur divers arbres fruitiers ont été observées. II s'agit de :

- Lasiodiplodia theobromae (Synonymes : Botryodiplodia theobromae, Lasiodiplodia tubericola)

- Fusarium solani

- $\quad$ Phoma glomerata

Tableau 1 : . Incidence relative des différents pathogènes isolés des plants malades

\begin{tabular}{l|c|c|c}
\hline Espèces fongique & $\begin{array}{c}\text { Nombre totale de plants } \\
\text { examinés }\end{array}$ & Nombre de plants positifs & Pourcentages \\
\hline Lasiodiplodia theobromae & 18 & 15 & 83,33 \\
Fusarium solani & 18 & 5 & 27,77 \\
Phoma glomerata & 3 & 16,66 \\
\hline
\end{tabular}

Tableau 2 : Incidences des trois champignons sur les différents sites

\begin{tabular}{l|c|c|c}
\hline Sites & $\begin{array}{c}\text { L. theobromae } \\
(\%)\end{array}$ & $\begin{array}{c}\text { F. solani } \\
(\%)\end{array}$ & $\begin{array}{c}\text { P. glomerata } \\
(\%)\end{array}$ \\
\hline Bassar & 71,43 & 14,29 & 0,00 \\
Kabou & 100,00 & 50,00 & 50,00 \\
Temèmè & 100,00 & 33,33 & 0,00 \\
Bandjeli & 100,00 & 0,00 & 100,00 \\
Sara & 66,67 & 33,33 & 0,00 \\
\hline
\end{tabular}


Tableau 3 : Incidences combinées des trois champignons sur les différents sites

\begin{tabular}{l|c|c|c|c}
\hline Sites & $\begin{array}{c}\text { L. theobromae + } \\
\text { F. solani } \\
(\%)\end{array}$ & $\begin{array}{c}\text { L. theobromae + } \\
\text { P. glomerata } \\
(\%)\end{array}$ & $\begin{array}{c}\text { F. solani + } \\
\text { P. glomerata } \\
(\%)\end{array}$ & $\begin{array}{c}\text { L. theobromae + } \\
\text { F. solani +P. glomerata } \\
(\%)\end{array}$ \\
\hline Bassar & 14,29 & 0,00 & 0,00 & 0,00 \\
Kabou & 50,00 & 50,00 & 25,00 & 25,00 \\
Temèmè & 33,33 & 0,00 & 0,00 & 0,00 \\
Bandjeli & 0,00 & 100,00 & 0,00 & 0,00 \\
Sara & 33,33 & 0,00 & 0,00 & 0,00 \\
\hline
\end{tabular}

Les isolats de $L$. theobromae ont été isolés des tissus malades provenant de $83.33 \%$ des échantillons de manguier $(66,67 \%)$ ou de citrus $(16,67 \%)$ sur tous les sites prospectés (tableau 1). Sur milieu artificiel mPDA (Fig.1. A), les colonies sont initialement blanches mais elles s'accroissent rapidement et deviennent très vite noires. Le mycélium est cloisonné et ramifié. A maturité on observe des pycnides à la surface des colonies. Les conidies sont initialement hyalins, unicellulaire et de forme oblong. Une fois mures elles laissent apparaitre une cloison centrale qui subdivise la conidie en 2 cellules. Les conidies de $L$. theobromae sont de couleur brun sombre, elles ont une paroi épaisse et leur surface externe présente des striations longitudinales. Le deuxième champignon isolé, Fusarium solani (Fig.1.B) a été observé dans $27.78 \%$ des échantillons (16,67 \% pour les manguiers et $11,11 \%$ pour les citrus). Ses colonies croissent rapidement. De couleur blanche généralement elles étaient teintées de jaune avec l'âge. Les macroconidies de $F$. solani sont composées de 3 à 5 cellules. Elles sont allongées et légèrement arquées.
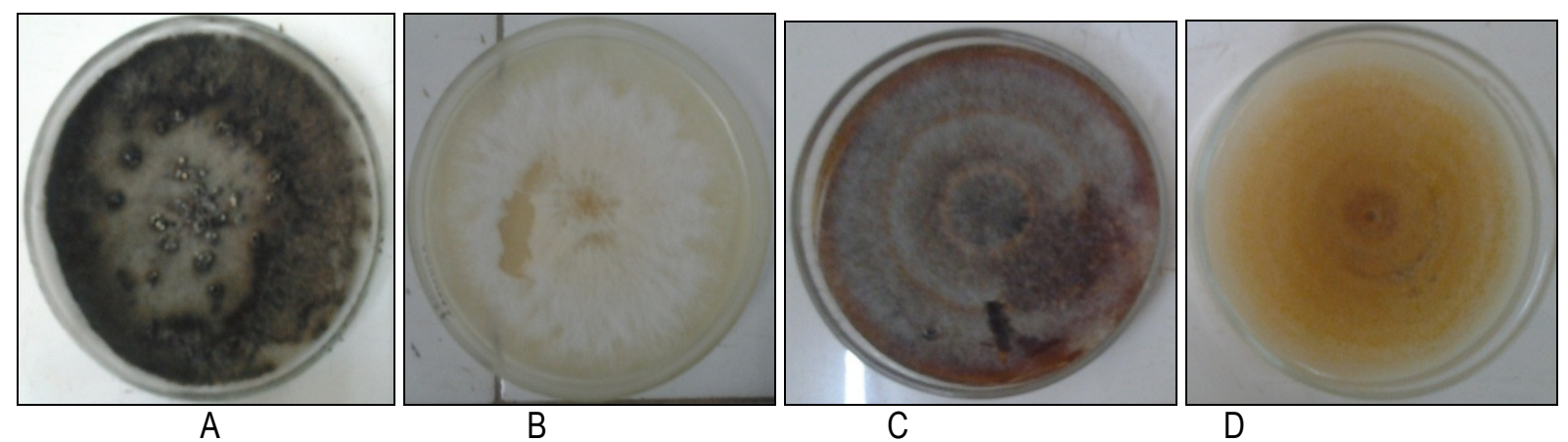

Fig. 1 : Colonie mûre de Lasiodiplodia theobromae (A) sur mPDA, de Fusarium solani sur PDA (B), et de Phoma glomerata sur mPDA (C) et PDA (D)

Enfin les isolats de Phoma glomerata (Fig. 1. C et D) ont été retrouvés sur seulement $16.67 \%$ des échantillons (11,11\% pour les manguiers et $5,56 \%)$. Ce champignon a aussi une croissance relativement rapide (maturité en 5 jours). Les colonies ont une texture veloutée et sont de couleur variant du brun au rose et à l'olivâtre. Le mycélium est segmenté et les conidies sont généralement unicellulaires de contenu clair à brun pale. Leur forme va de l'ovoïde à l'ellipsoïdale.
Test d'efficacité des fongicides : Au cours des huit jours d'évaluation à $31^{\circ} \mathrm{C}$ tous les témoins aspergés avec de l'eau distillée ont atteint le diamètre maximal des boîtes de Pétri c'est-à-dire les 9 centimètres. Dans le cas des traitements témoins sans aspersion d'eau, seules les colonies de $L$. theobromae ont atteint ce diamètre maximal alors que ceux de $F$. solani et de $P$. glomerata se sont limitées à un diamètre maximal de $6.69 \mathrm{~cm}$ et $6.01 \mathrm{~cm}$ respectivement (Fig.2). 


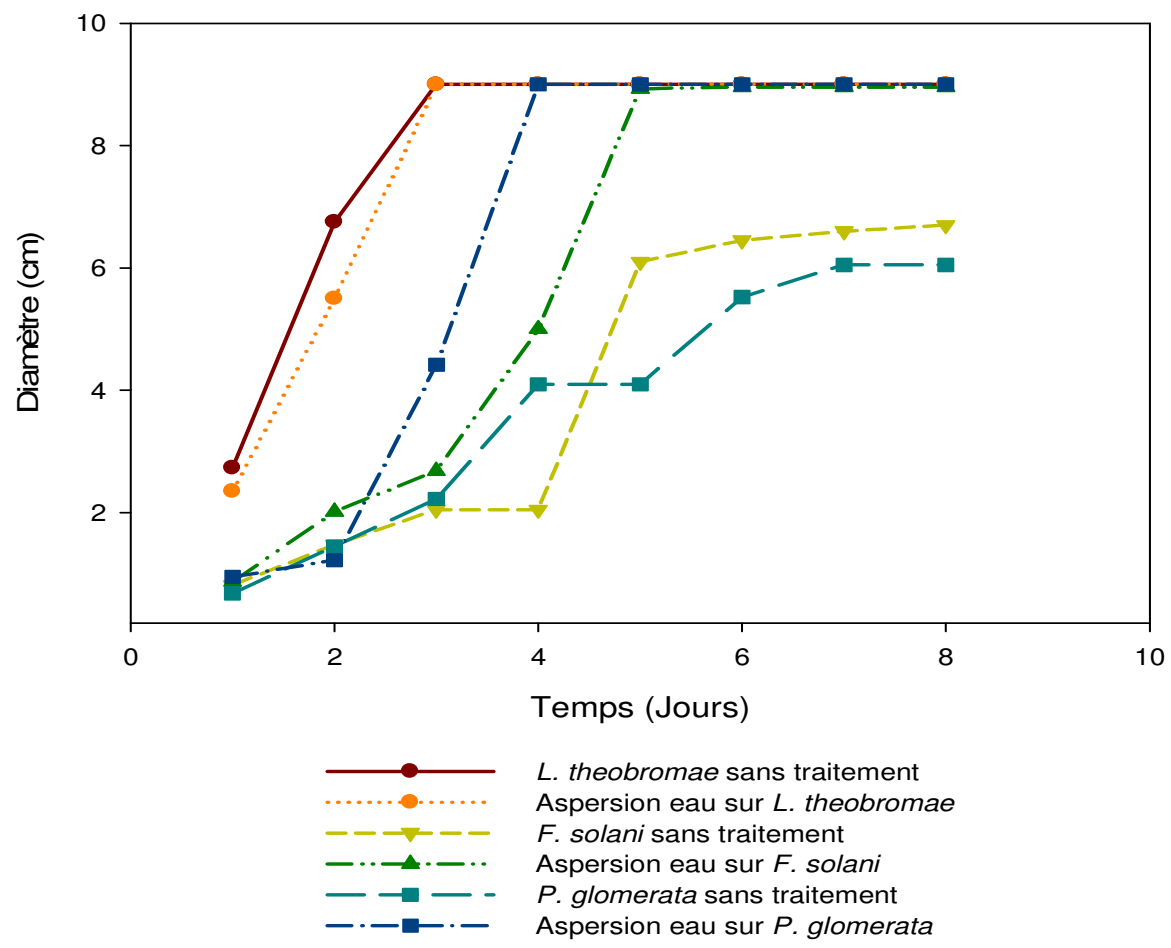

Fig. 2 : Croissance de $L$. theobromae, F. solani et $P$. glomerata sur milieu PDA sans traitement et avec aspersion d'eau distillée stérilisée

Les différents fongicides, dans un premier temps, ont eu des effets variables sur l'expansion des colonies de champignons. Dans un deuxième temps les méthodes d'apport des fongicides ont également eu des effets sensiblement différents et ceci quel que soit le fongicide ou le champignon. Dans tous les cas quand les fongicides sont incorporés au milieu (Fig. 3), on constate un effet important car les colonies ne croissent presque pas du tout, atteignant rarement 0,50, 1.8 et $1,11 \mathrm{~cm}$ pour $L$. theobromae, $F$. solani et $P$. glomerata respectivement (Tableau 4). Et il apparait que quelque soit le fongicide $L$. theobromae est presque complètement inhibé (Fig. 3). Dans les expériences consistant à une aspersion de suspension aqueuse de fongicide sur des colonies ayant déjà initié leur expansion, le diamètre maximum atteint, sans considération de la nature des fongicides, est de $9 \mathrm{~cm}$. Toutefois en considérant les fongicides individuellement, les réponses sont plus variées (Fig. 4). 


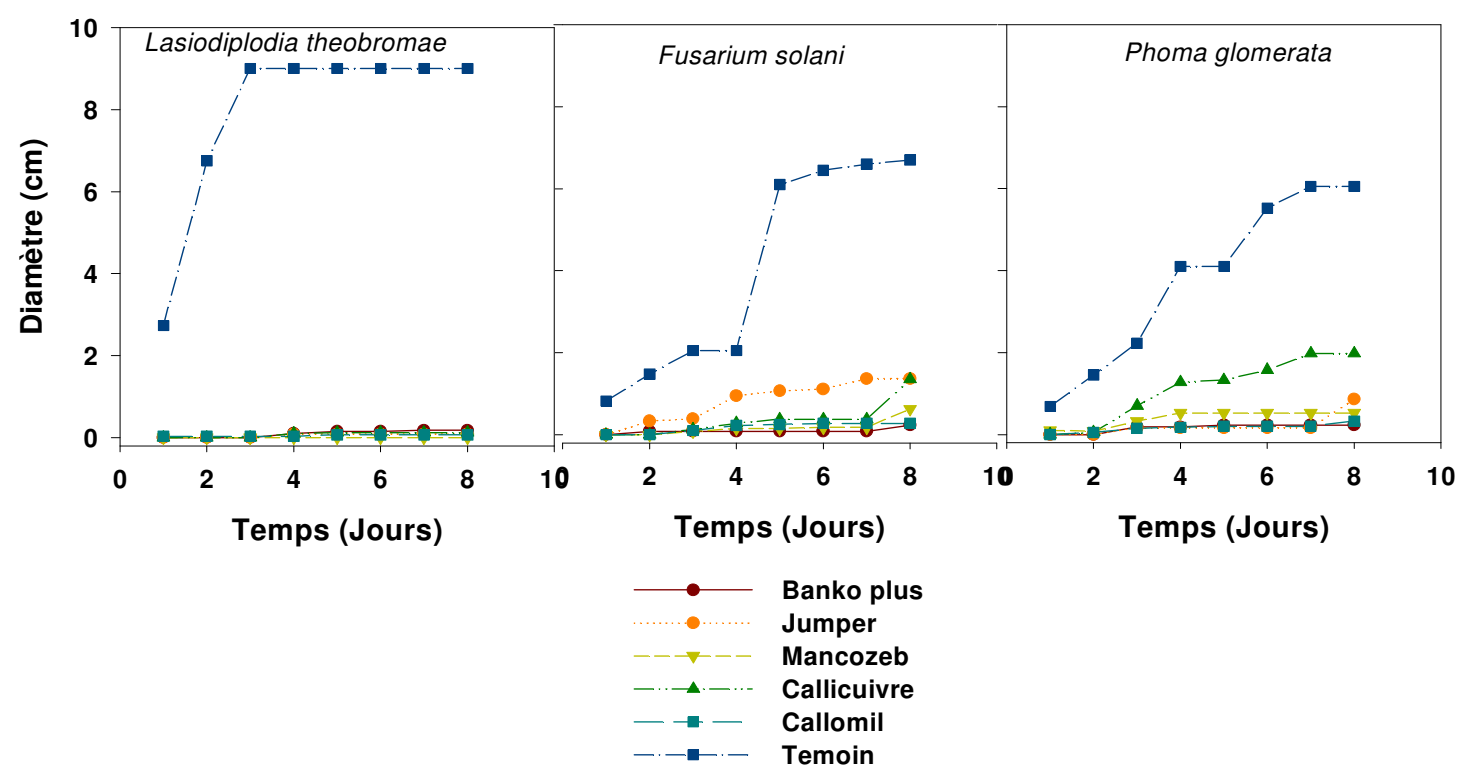

Fig. 3: Expansion des colonies de L. theobromae, F. solani et de $P$. glomerata en croissance sur le milieu avec incorporation directe des fongicides en comparaison avec le témoin absolu

Dans les conditions d'aspersion de suspension de fongicide sur les champignons après la germination des spores, pour L. theobromae, le Banko Plus donne le meilleur résultat alors que le Callomil est complètement inefficace (Tableau 4). Les trois autres fongicides se suivent dans l'ordre d'efficacité suivant: le Jumper, le
Mancozeb et le Callicuivre. F. solani est plus efficacement contenu par le Callicuivre et le Jumper suivi du Callomil, du Mancozeb et du Banco plus. Enfin le Banko plus, le Jumper, sont les fongicides les plus efficaces contre $P$. glomerata suivis dans l'ordre par le Mancozeb, le Callomil et du Callicuivre (Fig. 4).

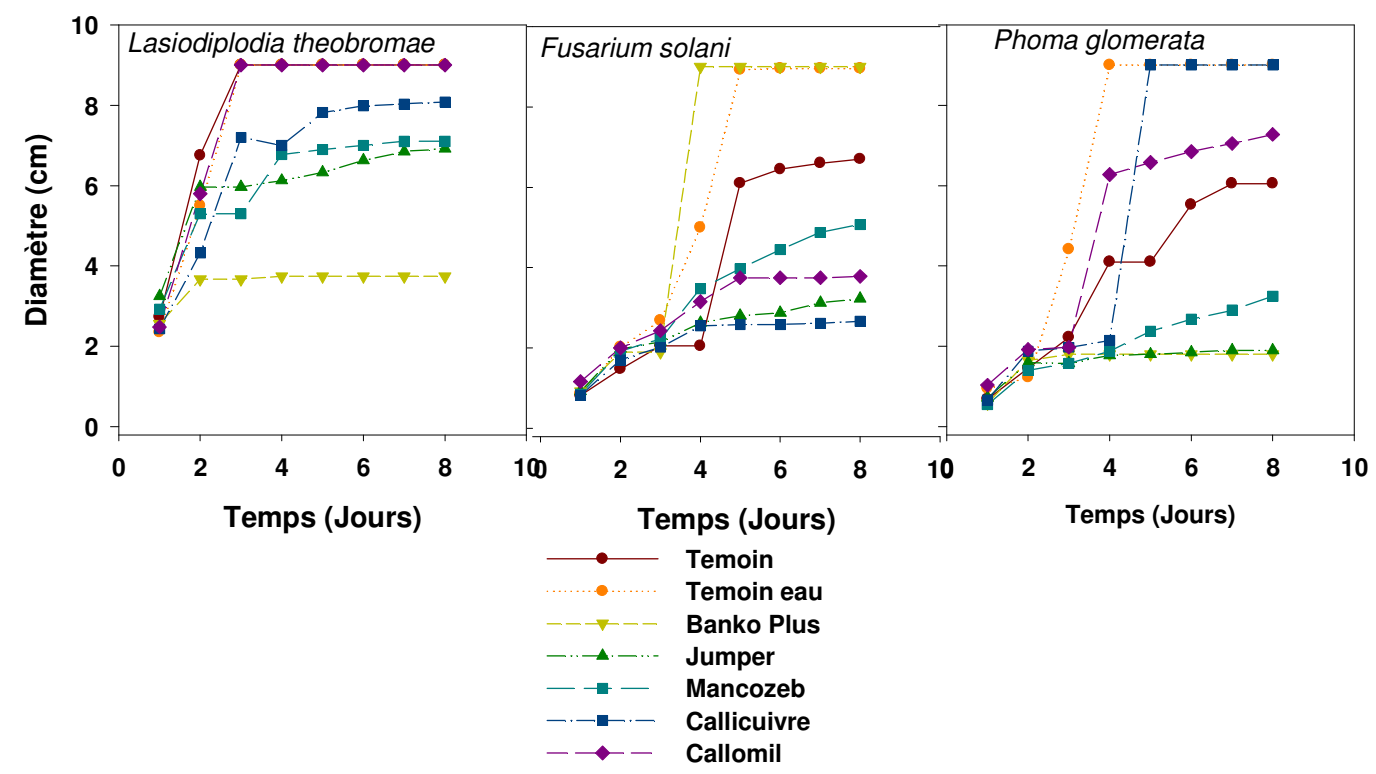

Fig. 4: Expansion des colonies de $L$. theobromae, F. solani et de $P$. glomerata en croissance sur du PDA après aspersion de suspension de fongicides 
Tedihou et al., J. Appl. Biosci. 2017 Dépérissement des manguiers et citrus dans la région centrale du Togo et méthodes de lutte par des fongicides

Tableau. 4: Moyennes globales des diamètres des colonies résultantes des traitements par les fongicides

\begin{tabular}{l|l|l|l|l|l|l}
\hline \multirow{2}{*}{ Fongicides } & \multicolumn{2}{|c|}{ Lasiodiplodia theobromae } & \multicolumn{2}{c|}{ Fusarium solani } & \multicolumn{2}{c}{ Phoma glomerata } \\
\cline { 2 - 7 } & IDM & ASE & IDM & ASE & IDM & ASE \\
\hline Témoin & $7,94 \mathrm{a}^{*}$ & $7,73 \mathrm{a}^{* *}$ & $4,03 \mathrm{~g}^{*}$ & $5,80 \mathrm{de}^{* *}$ & $3,77 \mathrm{gh}^{*}$ & $6,45 \mathrm{bc}^{* *}$ \\
Banko Plus & $0,09 \mathrm{p}$ & $3,58 \mathrm{gh}$ & $0,09 \mathrm{p}$ & $6,23 \mathrm{bcd}$ & $0,16 \mathrm{p}$ & $1,63 \mathrm{~lm}$ \\
Jumper & $0,05 \mathrm{p}$ & $6,00 \mathrm{~cd}$ & $0,82 \mathrm{no}$ & $2,46 \mathrm{jk}$ & $0,21 \mathrm{p}$ & $1,64 \mathrm{~lm}$ \\
Mancozeb & $0,50 \mathrm{p}$ & $6,05 \mathrm{~cd}$ & $0,17 \mathrm{p}$ & $3,36 \mathrm{hi}$ & $0,39 \mathrm{op}$ & $2,08 \mathrm{kl}$ \\
Callicuivre & $0,07 \mathrm{p}$ & $6,64 \mathrm{~b}$ & $0,36 \mathrm{op}$ & $2,19 \mathrm{k}$ & $1,11 \mathrm{mn}$ & $5,33 \mathrm{ef}$ \\
Callomil & $0,05 \mathrm{p}$ & $7,79 \mathrm{a}$ & $0,18 \mathrm{p}$ & $2,97 \mathrm{ij}$ & $0,16 \mathrm{p}$ & $4,87 \mathrm{f}$ \\
\hline
\end{tabular}

NB : Les moyennes avec la même lettre ne sont pas significativement différentes (LSD avec 0,05$) ;{ }^{*}$ : témoin absolu sans aucun traitement; ${ }^{* *}$ : témoin avec aspersion d'eau distillée stérile; IDM: Incorporation directe du fongicide dans le milieu de culture; ASE: Aspersion de la colonie avec une suspension du fongicide.

\section{DISCUSSION}

Les trois champignons pathogènes identifiés à la suite de ces travaux font supposer que ce n'est pas un seul champignon qui est responsable du dépérissement des fruitiers dans la zone de Bassar. Ce constat entraine une interrogation sur les interactions qui pourrait exister entre les virulences des trois pathogènes potentiels en présence sur les manguiers et les citrus malades. En effet la maladie pourrait résulter d'une synergie d'action des champignons pathogènes en présence ou juste de l'action d'un des trois champignons pathogènes, les autres étant présents de façon endophytique ou nécrophyte. Selon les résultats de deux études réalisées par Ko et al. (2004) et Khanzada et al. (2005), c'est la deuxième théorie qui est la plus plausible dans le cas de cette maladie de dépérissement. En effet plusieurs résultats ont montré qu'en présence concomitante de $L$. theobromae, avec d'autres champignons potentiellement pathogéniques, seul $L$. theobromae était l'agent causal de cette maladie de dépérissement (Ramos, 1997 et Saeed et al, 2011). En plus $L$. theobromae a été plus fréquemment retrouvé dans les tissus malades que les deux autres champignons. Les rares cas où il n'a pas été isolé des tissus malades pourraient être expliqués par, soit, la non présence du pathogène dans les tissus malades ou necrosés ou tout simplement par les limites de la technique d'isolation. II est donc juste de déduire que la réduction de l'impact du dépérissement des manguiers et des citrus devra passer par la lutte contre $L$. theobromae. L'incorporation du fongicide au milieu avant son inoculation avec le champignon a donné les meilleurs résultats. Dans ce cas les fongicides sont appliqués systématiquement avant la mise en contact de la plante et du pathogène. Ce traitement permet ainsi de juger de l'efficacité des fongicides dans l'absolu avant germination des spores. En effet tous les fongicides utilisés contiennent des principes actifs pouvant inhiber la germination des spores des champignons. Dans ces conditions, pour $L$. theobromae, champignon supposé responsable de la maladie, l'incorporation du Mancozeb a été la plus efficace suivi dans l'ordre par le Callomil, le Jumper, le Callicuivre et le Banko Plus. Cette variabilité est somme toute relative, parce que dans tous les cas, tous les fongicides ont été plus ou moins suffisamment efficace pour stopper ou empêcher la prolifération des champignons. Surtout que contrairement à l'étude similaire de Tovar et al. (2013) les doses étaient relativement élevées car mises pour respecter les directives d'utilisation du fabriquant. Cette situation pourrait être assimilable à une situation de traitement préventif de la maladie avant l'infection. Toutefois elle est difficilement réalisable dans la réalité surtout sur de vieilles plantes pérennes. Dans la réalité, les plantes sont en général traitées parce qu'on y a observé la maladie donc la maladie est déjà installée avant qu'on y apporte des remèdes. Pour prendre en compte cette réalité dans les conditions de laboratoire, les fongicides ne sont apportés sur les champignons, sous forme de suspension, qu'après leur germination sur le milieu de culture sans fongicide. Et dans ces conditions trois cas sont observés: ou bien la colonie cesse de croitre définitivement, ou bien sa croissance ralentie avant de reprendre plus tard mais en étant plus ou moins ralentie, ou encore elle continue son expansion sans réaction autre que celle correspondante à un apport d'eau. Dans ces conditions, pour $L$. theobromae, le Banko Plus donne le meilleur résultat. La nette efficacité comparative de Banko Plus peut s'expliquer par le fait que seul ce fongicide contenait du 
carbadazim qui est un benzimidazole systémique inhibant la croissance du mycélium. On peut donc en conclure que $L$. theobromae est beaucoup plus sensible au carbadazim et donc que le Banko Plus et d'autres fongicides contenant ce principe actifs à des dosages appropriés peuvent contrôler efficacement ce champignon dans les conditions de laboratoire. L'incorporation direct des fongicides dans le milieu de culture avant l'insémination du milieu se révèle très efficace surtout pour de grandes concentrations de fongicide. Dans le cas de dosages plus faibles tels qu'effectué dans les études de Denman et al. (2004) et de Tovar et al. (2013) cette incorporation directe permet d'aboutir à des efficacités comparées de différents fongicides et dans ces cas, de façon générale, les

\section{CONCLUSION}

La maladie de dépérissement des manguiers et des citrus sévissant dans la partie centrale du Togo est principalement causée par $L$. theobromae. Le fongicide binaire Banko Plus contenant du Carbadazim et du

\section{REMERCIEMENTS}

Cette étude a été possible grâce au soutien financier de I'Institut Togolais de Recherche Agronomique (ITRA) et de la Direction de la Protection des Végétaux (DPV). Les auteurs tiennent à remercier Dr. Bonfoh Bédibètè

\section{REFERENCES}

Al-Sadi AM, Al-Wehaibi AN, Al-Shariqi RM, AlHammadi MS, Al-Hosni IA, Al-Mahmooli IH et al-Ghaithi AG, 2013. Population genetic analysis reveals diversity in Lasiodiplodia species infecting date palm, Citrus, and mango in Oman and the UAE. Plant Disease 97:13631369.

Davis R M, Farrald C J, Davila D, 1987. Botryodiplodia trunk lesions in Texas citrus. Plant Dis. 71:848-849.

Denman S, Crous PW, Sadie A et Wingfield M J, 2004. Evaluation of fungicides for the control of Botryosphaeria protearumon Proteamagnificain the Western Cape Province of South Africa. Australasian Plant Pathology 33: 97-102.

Desprez-Loustau M-L, Marcais B, Nageleisen L-M et Piou D, 2006. Interaction effects of drought and pathogens in forest trees. Annals of Forest Science63: 597-612.

Haougui A, 2013. Dépérissement du manguier au Niger. Fiche technique numéro 615 . Niger: fongicides de contacte se révèlent moins efficaces que les fongicides systémiques. Dans les conditions de cette étude le fongicide contenant le principe actif systémique s'est révélé le plus efficace surtout dans le cas où le mycélium déjà présent y est exposé. Enfin, la combinaison suivante de fongicides peut être recommandée pour contenir le dépérissement des manguiers et des agrumes : II s'agit de la combinaison de Banko Plus et de Mancozeb. Cette combinaison prend en compte dans un premier temps l'aspect systémique et l'aspect "par contact " des différents principes actifs pour, dans un premier temps, ralentir la croissance du mycélium et ensuite bloquer de nouvelles germinations de spores.

chlorothalonil s'est révélé efficace in vitro pour lutter contre $L$. theobromae. Ce fongicide peut être utilisé en combinaison avec le Mancozeb pour lutter contre la maladie de dépérissement des manguiers et des citrus.

et Monsieur Gogovor Yawo Sefe pour leur supervision et Monsieur Hada Bassimbako pour son apport technique.

RECA, 1 p. disponible sur http://www.recaniger.org/spip.php?article615 Consulté le 12/5/2017.

Kausar P, ChohanS et Parveen R, 2009. Physiological studies on Lasiodiplodia theobromae and Fusarium solani, the cause of Shesham decline. Mycopath 7: 35-38.

Khanzada MA, Lodhi AM et Shaazad S, 2005. Chemical control of Lasiodiplodia theobromae, the causal agent of mango decline in Sidh. Pakistan Journal of Botany37: 1023-1030.

Khanzada MA, Rajput AQ et Shahzad S, 2006. Effect of medium, temperature, light and inorganic fertilizers on in vitro growth and sporulation of Lasiodiplodia theobromae isolated from mango. Pakistan Journal of Botany 38: 885889.

Khanzada MA, Lodhi AM et Shahzad S, 2004. Pathogenicity of Lasiodiplodia theobromae and Fusarium solani on mango. Pakistan Journal of Botany 36: 181-189. 
Ko WH, Wang IT et Ann PJ, 2004. Lasiodiplodia theobromae as a causal agent of kumquat dieback in Taiwan.Plant Disease 88: 1383.

Meah M B, Plumbley R A, Jeger M J, 1991. Growth and infectivity of Botryodiplodia theobromae causing stem-end rot of mango. Mycological Research 95:405-408.

Punithalingam E, 1976. CMI Descriptions of Pathogenic Fungi and Bacteria No. 519.Wallingford, UK: $\mathrm{CAB}$ International.

Ramos LJ, Lara SP, McMillan RT et Narayanan JR, 1997. The resistance of mango (Mangifera indica) cultivars to tip dieback disease in Florida. Plant Disease 81: 509-514.

Reckhaus $P, 1987$. Hendersonula dieback of mango in Niger. Plant Dis. 71:1045.

Saeed S, Khan M I et Masood A, 2011. Symptom development after artificial inoculation of botryodiplodia theobromae, a possible causal organism to quick decline in mango trees.Pakistan Journal of Agricultural Sciences 48: 289-294.

Slippers B, et Wingfield MJ, 2007. Botryosphaeriaceae as endophytes and latent pathogens of woody plants: diversity, ecology and impact. Fungal Biology Reviews 21: 90-106.

Tovar JM, Mora JA, Nava C, Téliz D, Villegas Á et Leyva SG, 2013. Control of Lasiodiplodia theobromae, the causal agent of dieback of sapote mamey [Pouteria sapota Jacq.) H. E. Moore and Stearn] Grafts in Mexico. Revista Fitotecnia Mexicana 36: 233 - 238. 